\title{
Gerechtigkeit, Medizin und Gesundheitspolitik
}

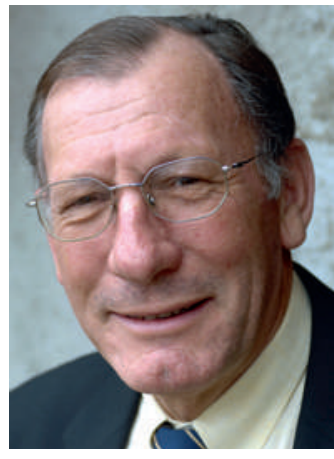

Jean Martin
Vom 26. bis zum 28. September 2012 fand in Tunis der «9th Global Summit of National Ethics Committees» statt, der unter der Ägide der WHO-Vertreter aus etwa vierzig Ländern aller Kontinente versammelte. Diskutiert wurde die Gerechtigkeit zwischen Menschen ein und desselben Landes und zwischen Ländern in unterschiedlichen materiellen und technologischen Entwicklungsstadien.

Der Hauptvortrag wurde von Professor Abdelwaheb Bouhdiba gehalten, dem bedeutenden Philosophen und Soziologen der Universität Tunis. Die Botschaften dieser Stimme des Mittelmeerraumes knüpfen auch an Sorgen in Westeuropa an.

«Es ist der Übergang von der Gerechtigkeit zum Recht, der Sorgen bereitet [...]. Ein gerechtes Urteil würde also nicht unbedingt auf der strikten Anwendung von Gesetzen und noch weniger auf der exakten Umsetzung von Regelwerken basieren. Die Gerechtigkeit führt das Gesetz, gibt die Ausrichtung vor, korrigiert. Sie rückt die Gesetze näher an das Recht» (übergeordnet, im Sinne der Menschenrechte), sagte Bouhdiba. Das ist richtig: Das Gesetz ist der unabdingbare Rahmen für das Zusammenleben in der Gesellschaft. Es gibt vor, was zulässig ist und was nicht und was demzufolge bestraft wird. Das Gesetz hat nichts mit Empathie oder harmonischem Zusammenleben zu tun.

Zur Politik: Es ist bekannt, dass sie, selbst wenn man lieber nichts mit ihr zu tun haben möchte, doch immer wieder mit einem selbst zu tun hat: «Die Politik - und infolgedessen auch die Gesundheitspolitik - ist eine unendliche Aneinanderreihung von Kompromissen. Sie entscheidet, welchen Platz Gerechtigkeit und materielle Anpassungen einnehmen und wie Güter und Vorteile verteilt werden sollen. Bei dieser gigantischen (Um-)Verteilung kommt dem gerechten Staat die Rolle zu, die Minderbegünstigten zu begünstigen, ohne dabei die Begünstigten zu benachteiligen.» Bezüglich der letzten Zielsetzung stellt sich die Frage, ob Bouhdiba nicht zu idealistisch argumentiert. Beim Management kollektiver Haushalte ist dem einen kaum etwas zu geben, ohne dem anderen etwas zu nehmen ...

Es gibt Verhandlungen, die Geduld erfordern: «Was gerecht ist, sollte gesetzeskonform sein, doch da das Recht im Zentrum aller widersprüchlichen Forderungen einer Gesellschaft angesiedelt ist, muss es zwischen den verschiedensten Kriterien abwägen. Letztlich läuft alles auf einen Kompromiss hinaus, geprägt von Zugeständnissen [...]. Daher diese gezwungenermassen ungleichen, instabilen Verteilungsvorgänge.» Schlechte Kompromisse sind häufig nur die weniger schlechten Lösungen. «Gesellschaften sind immer nur übergangsweise gerecht.»

Starke Worte: «Es gibt keine gute Gesundheitspolitik ohne eine gesunde Politik.» Hier besteht eine Verbindung zu dem, was ich zu vermitteln suche, wenn ich von Gesundheitspolitik sprechen soll. Für die Politik gilt das Salus publica suprema lex, was zwangsweise mit dem Salus aegroti suprema lex des Arztes, der sich um das Wohl des individuellen Patienten kümmert, kollidiert - ein Spannungsverhältnis, mit dem jeder nach bestem Wissen und Gewissen umzugehen hat. Auch ein zweites Ideal ist komplex: Es sollte weniger auf eine öffentliche Gesundheitspolitik (public health policy) als vielmehr auf eine gesunde öffentliche Politik (healthy public policy) gesetzt werden, eine Politik, die Gesundheit durch multi- und intersektorielles Handeln aller am gesellschaftlichen Leben Beteiligten und der Regierung fördert. Nahezu alle Bereiche menschlichen Lebens wirken sich auf die Gesundheit aus, entweder indem sie sie unterstützen oder ihr Schaden zufügen.

Bezüglich der Alltagszwänge setzte der Redner Akzente, die auch bei uns häufig zu hören sind: «Die Medizin hat sich ohne Zweifel verändert. Sie wurde industrialisiert, informatisiert, und das «Big Business» hat in ihr Einzug gehalten. Mit der Medizin von gestern hat sie kaum noch etwas zu tun [...]. Das wünschenswerte Gleichgewicht in der Beziehung zwischen Arzt und Patient ging in einem Netzwerk anonymer Verflechtungen mit Verwaltung, Kassen und Pharmaindustrie verloren. Pflege wurde zum Massenobjekt, Behandlungen zur Fliessbandarbeit.» Vergleichbare Probleme in ganz anderen Kontexten!

Pragmatismus: «Gerechtigkeit steht nicht für eine arithmetische Gleichheit; sie sorgt vielmehr dafür, dass die Rechte und Pflichten des Bürgers konkreten Lebenssituationen angepasst werden. Es gibt keine Standardlösungen. Gerechtigkeit ist nicht abstrakt. Sie ist jeweils situationsbezogen [1]. «Ein gerechtes Gesundheitssystem», resümiert Bouhdiba, «setzt eine soziale Solidarität voraus (welche die Prioritätenordnung definiert), ein effizientes Management und eine Humanisierung, die jene Zwischenräume gewährleistet, durch die sich Gerechtigkeit praktizieren lässt.» Die Arbeit in den Zwischenräumen, den Randbereichen, führt häufig systemunabhängig zu jenem zusätzlichen Quäntchen Qualität (Seele?), das für die therapeutische Beziehung erforderlich ist.

Jean Martin, Mitglied der Nationalen Ethikkommission und der Redaktion 